\title{
Magnetization reversal behavior in complex shaped Co nanowires: a nanomagnet morphology optimization
}

\author{
Fatih Zighem ${ }^{*}$ and Silvana Mercone $\dagger^{\dagger}$ \\ Laboratoire des Sciences des Procédés et des Matériaux, \\ CNRS-Université Paris XIII, Sorbonne Paris Cité, Villetaneuse, France
}

(Dated: October $22^{\text {nd }}, 2014$ )

\begin{abstract}
A systematic micromagnetic study of the morphological characteristic effects over the magnetic static properties of Co-based complex shaped nanowires is presented. The relevance of each characteristic size (i.e. length $L$, diameter $d$, and size of the nanowires head $T$ ) and their critical values are discussed in the coercive field optimization goal. Our results strongly confirms that once the aspect ratio $\left(\frac{L}{d}\right)$ of the nanowire is bigger than around 10 , the length is no more the pertinent parameter and instead the internal diameter and the shape of the nanowires play a key role. We attribute this behavior to the non uniform distribution of the demagnetizing field which is localized in the nanowires head and acts as a nucleation point for the incoherent magnetization reversal. Finally, angular dependence of the magnetization are simulated and compared to the case of a prolate spheroid for all considered morphologies.

PACS numbers:

Keywords: micromagnetic simulations, magnetic nano-objects, magnetization curves
\end{abstract}

\section{INTRODUCTION}

Magnetic one-dimensional nanowires have shown peculiar properties interesting for a bunch of different possible applications. Among them it can be mentioned the high density magnetic recording[1], the microwave devices[2] and also a new generation of permanent magnets $[3$, 4. In this latter case the nanowire can constitute the nanomagnet itself inside the surrounding media or it can also be the building block of dense nanostructured magnet bulk systems. In both cases the key issue is to maximize the magnet performances at a nanoscale level (i.e. high remanent magnetization and large coercive field). The most common approach to solve this issue, is to use non-spherical nanoparticles as they may naturally display a large shape anisotropy [5, 6] added to the magnetocrystalline one and thus easily beat the unwished superparamagnetic behavior usually occurring for nanosized particles at room temperature [1]. The basic idea is then to maximize the effective magnetic anisotropies (both shape and magnetocrystalline ones) in ferromagnetic systems (i.e. Co and $\mathrm{Fe}$ based for instance). In this context, coercive fields above $10 \mathrm{kOe}$ and remanent to saturation magnetization ratio near 1 are expected in single crystal cobalt and iron based nanorods. Several ways have been developed for the elaboration of such nano-objects: i) templates methods which involve the growth inside a host nanoporous matrix of nanorods or nanowires usually by electrochemical deposition [7[10] and ii) alternative chemical ones involving their synthesis in a solution. The soft chemistry route presents several advantages compared to the electrochemical one

*Electronic address: zighem@univ-paris13.fr

${ }^{\dagger}$ Electronic address: mercone@univ-paris13.fr
[11 15]: the low coast price, the relatively low synthesis temperature, the high crystalline quality and pureness of the chemical phase and also the good dispersion of the nanowires. On the other hand, despite this good control, the nanowires obtained by the chemical route usually show complex shaped one-dimensional morphology [12, 17, 18 and have never shown the expected theoretical coercive field value 3, 19. Previous theoretical studies tried to explain the observed gap by focusing on how the effective magnetic anisotropy can be mainly decreased both by complex shape of the nanowires and by a nonsingle crystal structure. At its turn, this change in the effective anisotropy modify significantly the magnetization reversal processes and consequently affects the magnetic properties wished for applications [20 22]. Those previous results highlighted, on the one hand, the importance of crystal structure uniformity (i.e. absence of stacking faults) in order not to locally decrease the magnetocrystalline anisotropy coefficient along the nanowire. On the other hand, they also shown that different morphologies can induce very unwished magnetic coercivity meltdown. This latter has been attributed to the presence of a demagnetizing field distribution at the edge of the nanowires which seems to be strictly linked to their complex shape and to let the reversal mechanism happening at lower coercive field. Unexpectedly, the demagnetizing values were found to be very high both in non-complex shaped nanowires (i.e. cylinder type) presenting a high coercive behavior then in complex shaped ones having a very modest coercivity. All those previous studies put in evidence the still open question of the understanding of the magnetization reversal mechanism in nanowires systems as well as the presence of concomitant behaviors that can act contradictorily for the optimization of the nanowires magnetic properties [23]. For example, it is easy to understand that a long nanowire giving rise to a high aspect ratio (length of nanowire versus diameter 


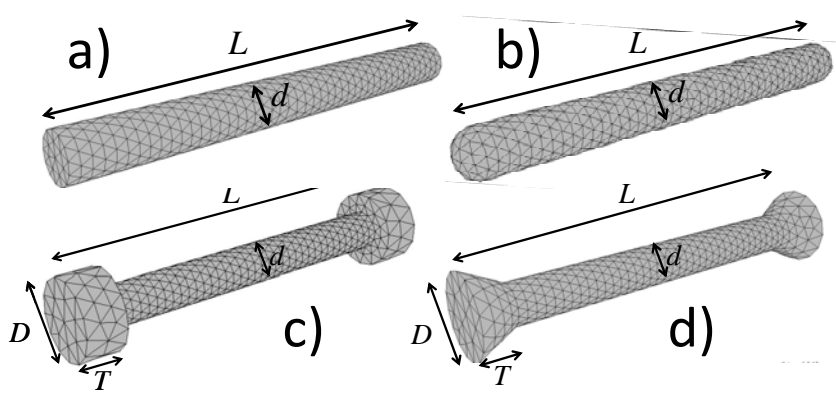

Figure 1: Typical meshes of the different considered nanoobjects: a) cylinder, b) capped cylinder, c) dumbbell and d) diabolo). The length of these nano-objects is $L=100$ $\mathrm{nm}$ while the internal diameter is $d=10 \mathrm{~nm}$. Moreover, the parameters $D$ and $T$ are equal to $20 \mathrm{~nm}$ and $10 \mathrm{~nm}$, respectively. Note that the distance between two nodes is smaller then $4 \mathrm{~nm}$ in all the simulated meshes.

in the middle of the nanowire) is needed for the optimization of the shape anisotropy coefficient while a very long nanowire increases dramatically the probability of a high density of stacking faults (low uniformity of the nanostructure) and consequently improve the probability of decreasing the magnetocrystalline effective coefficient. Thus, if the general idea coming up from a vast landscape of experimental and theoretical studies, is that a long nanowire will improve the effective magnetic anisotropy, it also goes in the other way. Thus, the issue concerning the compromise to reach between the contradictory effects coming from structure and morphology optimization is still relevant.

Thereby, a systematic study of the morphological characteristics effects over the magnetic static properties of Co-based complex shaped nanowires is proposed here. Starting from the experimental shapes observed, we performed a micromagnetic study of the reversal magnetization mechanism varying continuously the nanowire geometry. The relevance of each characteristic size (i.e. length, diameter and size of the head) and their critical values are discussed in the coercive field optimization goal.

\section{MICROMAGNETIC METHOD}

The micromagnetic calculations have been performed by using the NMAG micromagnetic simulation package [25. This software is indeed well suited to describe the complex shape of the nano-objects fabricated thanks to the polyol process as it allows to simulate very different shapes related to the one-dimensional simple nanowire. The magnetic parameters of all the studied nano-objects correspond to typical values of cobalt material 24. Thus the saturation magnetization $\left(M_{S}=1.4 \times 10^{3} \mathrm{emu} . \mathrm{cm}^{-3}\right)$ and the exchange stiffness $\left(A=1.2 \times 10^{-6} \mathrm{erg} . \mathrm{cm}^{-1}\right)$ have been defined from the bulk reference. In order to dissociate the effect of the morphology from the one coming from the structure, we considered a single crystal nanowire (i.e. the magnetocrystalline anisotropy is kept constant and in our case equal to zero). In this condition, an exchange length $\left(\ell_{e x}=\sqrt{A / 2 \pi M_{S}^{2}}\right)$ around $3.2 \mathrm{~nm}$ is then deduced. Once defined the magnetic properties, an important step of the procedure consists in defining the objects geometries and to their discretization by using a mesher (NETGEN and GMSH in the present case [26, 27]). Figure 1 presents the typical nano-objects which will be considered throughout this work, namely: a) a cylinder, b) a capped cylinder, c) a dumbbell and c) a diabolo. Thereafter, $z$-direction (see Figure 1) will corresponds to the revolution axis of the studied objects (considered parallel to the length of the nanowires). All these nanoobjects are characterized by a length $L$ and a diameter in the middle $d$. Two more geometrical parameters are necessary for the diabolo and the dumbbell morphology study: the tip width $D$ and the tip thickness $T$. We compared the results obtained for these specific nanoobjects to the well-known standard geometry of a prolate spheroid (ellipsoid) [28]. The length $L$ of our nanowires varies from $50 \mathrm{~nm}$ to $2000 \mathrm{~nm}$ while the internal diameter vary from 6 to $20 \mathrm{~nm}$. Typical length and diameters experimentally observed by using the polyol process elaboration are within these ranges. It is important to underline here that the distance between two nodes of the different meshes used in all the simulated geometries, is most of the time smaller than $\ell_{e x}$. Finally, the NMAG package is employed to solve the Landau-Lifshtiz-Gilbert equation. As only the static magnetization configurations are examined in all the performed simulations, the damping Gilbert constant $(\alpha)$ has been set to 0.5 in order to minimize the computing time. The magnetization reversal mechanism of the different nanowires has been studied mainly throughout the magnetization curves calculations along $z$ direction. The magnetization curves are investigated by applying an external magnetic field $\vec{H}$ ranging from $-10 \mathrm{kOe}$ to $+10 \mathrm{kOe}$; it is incremented by steps of about 50 Oe.

\section{RESULTS AND DISCUSSION}

As previously mentioned, one of the still open question that we addressed here, is the range of the length size of the nanowires playing a role on the static magnetic properties of these latter. As already detailed in the introduction, there is an important compromise to reach between a long nanowire and a perfect crystallized one. In this purpose we address here the question of the minimum limit for the nanowire length, necessary for a given geometry to optimize the shape anisotropy coefficient. The ellipsoidal (prolate spheroid) nano-objects will be used as reference because their well known static magnetic behavior set the upper limit of the shape anisotropy coefficient for a given length $(L)$ and internal diameter (d) 6]. In this latter standard geometry, two of the three axis have the same length and the third one is bigger than the two others. In this configuration, the coercive 


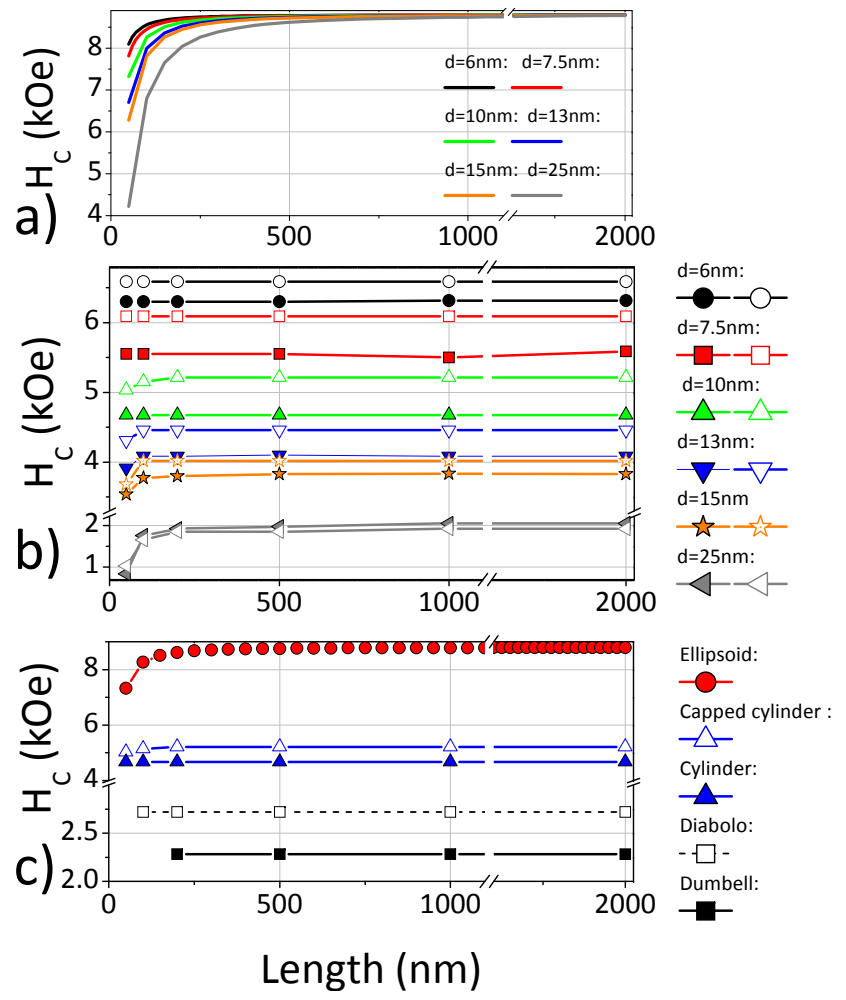

Figure 2: Coercive field along the revolution axis as function of the length $L$ for: a) prolate spheroid objects with different aspect ratios b) cylinder (filled symbols) and capped cylinder (open symbols) nanowires. c) Comparison of the coercive field among the various shaped objects as function of the length $L$ and for a diameter in the middle $d=10 \mathrm{~nm}$. The parameters $D$ and $T$ for the dumbbell and the diabolo nanowires have been fixed to $20 \mathrm{~nm}$ and $10 \mathrm{~nm}$ respectively.

field $H_{C}$ along the revolution axis is simply given by [28]:

$$
H_{C}=4 \pi\left(N_{z}-N_{x}\right)
$$

where $N_{z}$ and $N_{x}$ (and $N_{y}$ ) are the demagnetizing factors which can be analytically determined by using the following relations [28]:

$$
N_{x}+N_{y}+N_{z}=1
$$

$$
N_{z}=\frac{1}{a^{2}-1}\left(\frac{a}{2\left(a^{2}-1\right)^{0.5}} \log \left(\frac{a+\left(a^{2}-1\right)^{0.5}}{a-\left(a^{2}-1\right)^{0.5}}\right)-1\right)
$$

where $a$ is the aspect ratio $(a=L / d)$. Figure $2 a)$ presents the calculated coercive field of ellipsoidal nanoobject for various diameters $(d)$ ranging from 6 to 25 $\mathrm{nm}$ and as function of $L$ (i. e. aspect ratio varying from 2 to 330) by using equations 1 and 3 . It clearly appears that $H_{C}$ tends to the value of an infinite cylin$\operatorname{der}\left(H_{C}=2 \pi M_{S}=8800\right.$ Oe $)$ for all the diameter when $L$ increases. This tendency, as expected, is more and more efficient as the length over internal diameter ratio increases. At small aspect ratios (below $L=500 \mathrm{~nm}$ ), a non-negligible $H_{C}$-variation is observed, especially for the higher considered diameters. For instance, for $d=25$ $\mathrm{nm}$ (resp. $d=6 \mathrm{~nm}$ ), the coercive field varies from 4.2 $\mathrm{kOe}$ (resp. $8 \mathrm{kOe}$ ) to $8.6 \mathrm{kOe}$ (resp. $8.8 \mathrm{kOe}$ ) for a length varying from 50 to $500 \mathrm{~nm}$. The observed behavior points out that whatever is the size of $L$ and $d$, once the aspect ratio is bigger than around 10 the coercive field reaches the maximum. This is confirmed also by all the others shaped nano-objects (see Figure 2c)). Thus, in case of one-dimensional nano-objects for typical diameter $d$ around $10 \mathrm{~nm}$, a minimum length of $100 \mathrm{~nm}$ is sufficient to reach the maximum coercive field value. All the experimental effort focusing on improving this aspect ratio value by extending the length of nanowires at the fixed $d$, will be useless in term of shape anisotropy optimization [19]. This is true whatever is the morphology of the nanowires. Another interesting feature in Figure 2 $\mathrm{b}$ ) is that the coercive field variations calculated by micromagnetic simulations on capped cylinders and cylinders nanowires of similar aspect ratios have a different behavior. The most important difference is that the calculated $H_{C}$ values strongly depend on the diameter $d$ while it remains constant in almost the whole $L$ range. Thus in a large range of length $(L=100-2000 \mathrm{~nm})$ the smaller is the internal diameter $d$, the higher is the coercive field. This result strongly confirms that once the aspect ratio $\left(\frac{L}{d}\right)$ of the nanowire is bigger then 10, the length is no more the pertinent parameter and instead the internal diameter and the shape (cylinder or capped cylinder) of the nanowires play a key role. The shape effect is strongly confirmed by the diabolos and dumbbells nano-objects behavior presented in Figure 22). In this graph, a comparison in between all the four discussed morphologies is performed for an internal diameter of $10 \mathrm{~nm}$. The parameters $D$ and $T$ of the dumbbells and the diabolos tips have been fixed to $20 \mathrm{~nm}$ and $10 \mathrm{~nm}$, respectively. Impressively, nanowires with the same aspect ratio, show very different coercive behavior. This confirms the importance of the edge morphology over the magnetic shape anisotropy efficiency. In order to analyze this effect, we focused our attention on the demagnetizing field distribution inside the nanowires.

As previously reported [6, 22], for a prolate spheroid (ellipsoidal nano-object) the demagnetizing field is uniform and equal to $\vec{H}_{d e m}=-4 \pi \overline{\bar{N}} \vec{M}$. Figure 3 shows the demagnetizing field distributions (along the revolution axis) at a saturating magnetic field applied (i.e. the magnetic moments are aligned along this $z$ axis). These distributions have been calculated for nano-objects of 200 $\mathrm{nm}$ in length and $10 \mathrm{~nm}$ in diameter (i.e. $\frac{L}{d}>10$ ). It is worth mentioning that demagnetizing field distributions and nucleation volume variations during the reversal process have been largely studied in literature in individual magnetic bits of different geometry (prism, sphere and cylinder one) (i.e. $\frac{L}{d}<10$ ) and thus will be not reconsidered here [6, 29]. Also it should be underlined that in our case, because of the uniform distributions of the mag- 


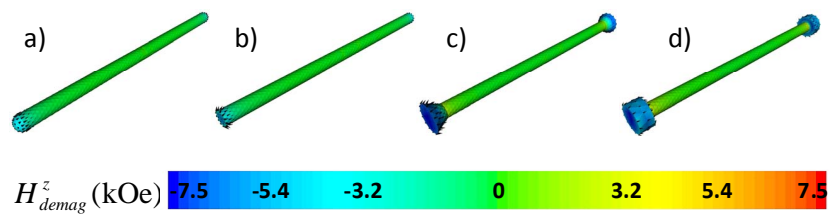

Figure 3: Demagnetizing field distributions inside the different nano-objects at a saturating field: a) capped cylinder, b) cylinder, c) dumbbell and d) diabolo). The geometrical parameters are fixed as follows: $L=200 \mathrm{~nm} ; d=10 \mathrm{~nm}$ ( $D=20 \mathrm{~nm}$ and $T=10 \mathrm{~nm}$ for the dumbbell and the diabolo). The colors encode the $z$-component of the demagnetizing field $H_{\text {dem }}$ ( $x$ - and $y$-components are close to zero in all the presented cases).

netic moments, only the $z$-component of $\vec{H}_{d e m}$ are non zero (see color map of Figure 3). More precisely, the demagnetizing field is almost zero in the whole nano-objects except at the tips where values in the range 4.0-7.5 kOe can be reached. The higher values are obtained for the diabolo and dumbbell shapes (around $7.5 \mathrm{kOe}$ for the maximum value) whereas smaller values are obtained in the capped cylinder and cylinder shapes (around $4 \mathrm{kOe}$ for the maximum value). Similar $\vec{H}_{d e m}$-distributions are obtained whatever the value of the length $L$ (above 100 $\mathrm{nm}$ ) which again means that once the aspect ratio is fixed, these distributions are the key parameter in the nucleation of the magnetization reversal mechanism. In order to study into details this shape effect, we decided to compare the hysteresis loops of the four different shapes discussed.

Looking at Figure 4a), the magnetization curves calculated along the revolution axis for the different types of nano-objects, we can separate them into two groups. Indeed, two hysteresis loops shapes are recognized: on the one hand the cylinder and capped cylinder nano-objects hysteresis behavior and on the other hand the dumbbell and diabolo nano-objects ones. While the hysteresis loops of the cylinders and capped cylinders are relatively squares (for applied field along the revolution axis), the ones of diabolos and dumbbells clearly presents a linear dependence before the reversal which is characteristics of a non uniform magnetization distribution. Figures 4b) and c) present the magnetization distributions and the corresponding demagnetizing field distributions inside the cylinder and the diabolo during the reversal process. The distributions of the capped cylinder and the dumbbell objects are not shown here, as their configurations have been found close to the ones of the cylinder and the diabolo, respectively. The magnetization distribution is almost uniform during the whole reversal process for the cylinder whereas it presents non-negligible $x$ - and $y$-components at the tips during the reversal mechanism in the diabolo case. The linear decreasing dependencies of $\frac{M_{R}}{M_{S}}$ ratios during the reversal mechanism for the second group of nano-objects, are clearly due to these non uniform magnetization distribution. In
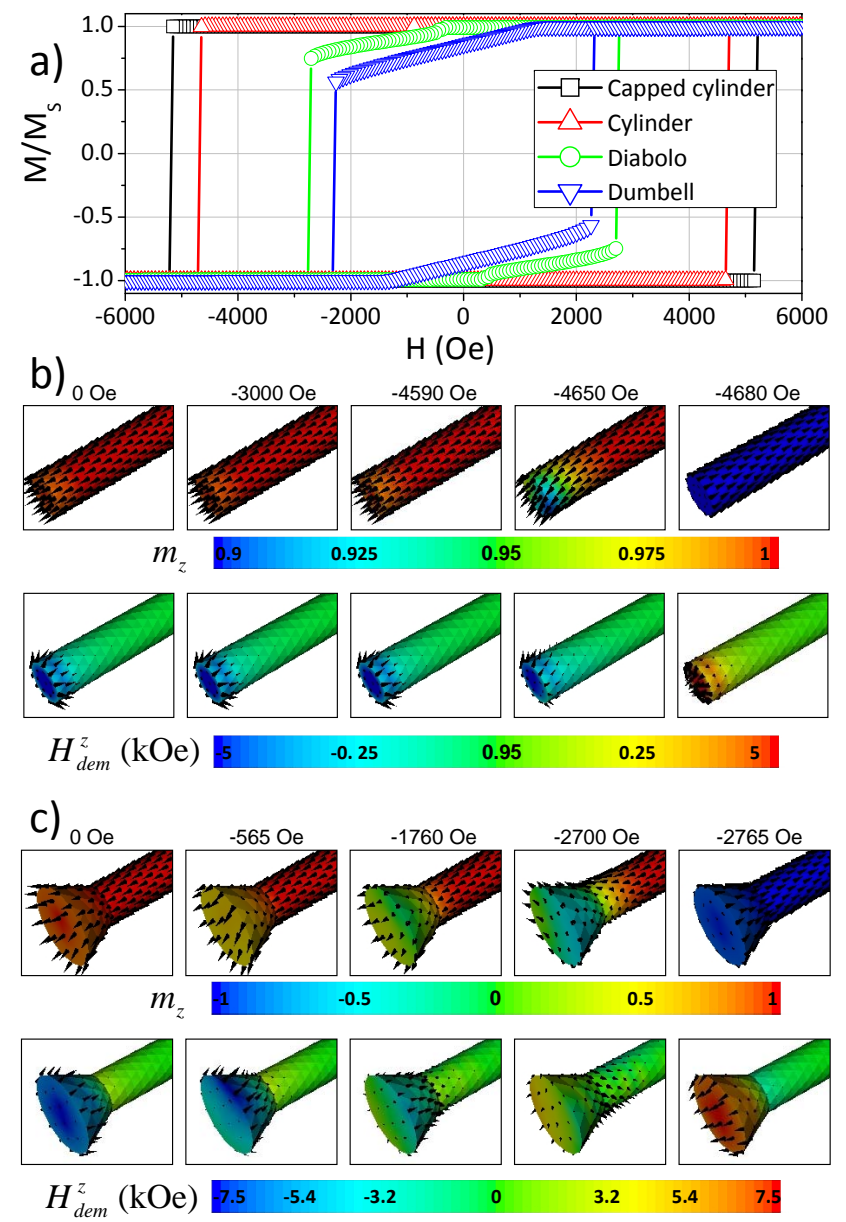

Figure 4: a) Magnetization curves calculated along the revolution axis for the different types of nano-objects. b)c) Magnetization (up images) and demagnetizing field (down images) distributions inside the cylinder (b) and the diabolo (c) during the reversal process. The geometrical parameters are fixed as follows: $L=200 \mathrm{~nm} ; d=10 \mathrm{~nm}(D=20 \mathrm{~nm}$ and $T=10 \mathrm{~nm}$ for the dumbbell and the diabolo)

this case the magnetization remains uniform only before $(H<500$ Oe $)$ and after $(H \sim-2800$ Oe $)$ the reversal process. Comparing the demagnetizing field of these two groups of nano-objects in the magnetic state where the magnetization is uniform, it is interesting to note that lowest coercive fields are obtained for nano-objects presenting the higher localized demagnetizing field (i. e. dumbbell and diabolo). In both cases demagnetizing field is localized at the tip and is very high while the magnetization is uniform (i.e. before and after the reversal). During the reversal mechanism (see Figure 4 r)), it is clear that the demagnetizing field can reach very low values (close to zero) even for the second group of nanoobjects. All those results bring to the conclusion that the tip play the role of nucleation point for the reversal of the magnetization. Figure 4 ) also shows that the $\frac{M_{R}}{M_{S}}$ ratios are lower for the dumbbell and diabolo nanowires, as already stressed out. This ratio is dependent of the 


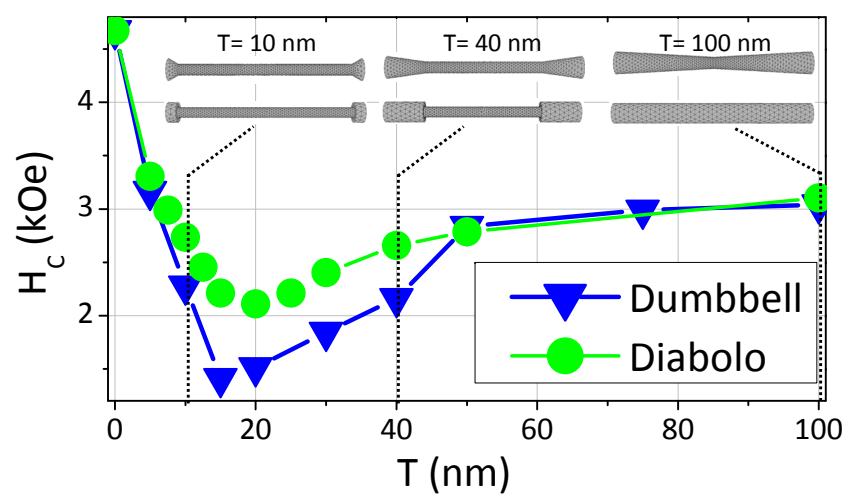

Figure 5: Coercive field values for dumbbell and diabolo nanowires with an aspect ratio $\frac{L}{d}=20$ (i.e. $L=200 \mathrm{~nm}$, $d=10 \mathrm{~nm}$ and $D=20 \mathrm{~nm}$ ) as function of the thickness $T$ of the edge; meshes of the nanowires for 3 different thickness are shown in the upper part of the figure.

volume of the nano-objects, so it is possible to increase them by increasing the length of the nano-objects. For this shaped nano-objects then, increasing the $\frac{M_{R}}{M_{S}}$ ratios would be the only reason for an elaboration effort towards longer nano-objects while either for the coercivity optimization as stated before and for thermal stability improvement 29, this effort would be useless. In order to go into details of the nucleation influence of the demagnetizing field on the magnetization reversal mechanism, we studied the coercive behavior of the dumbbell and diabolo nano-objects as function of the thickness $T$ of the edge.

Figure 5 shows very clearly a meltdown of the coercive behavior of both the dumbbell and diabolo nanowires during the increasing of $T$ value. Interestingly a critical thickness around $T \sim \frac{L}{d}$ is observed for the minimum value of the coercive field in both the geometry. Once this minimum reached, the coercive field goes, for both dumbbell and diabolo nanowires, towards the coercive field value of the cylinder type nanowires having the aspect ratio of 20 (see Figure 2b)). This is intuitively understood by the tendency of the nanowires shape (both for dumbbell and diabolo-see Figure 2b)) to resemble more and more, with the increasing of the head thickness $T$, to a cylinder type nano-object. This result is coherent with previous ones stressing out the key role of the tips as nucleation points for the magnetization reversal mechanism (i.e. melting down of the coercivity as function of the increasing of $T$ value). Even more, the presence of a critical thickness for the tip above which the coercivity increases again to reach the cylinder value, highlights the morphology importance on the shape anisotropy optimization.

In order to study the effect of the morphology on the nanowires behavior under the application of a magnetic field, we performed the micromagnetic simulation of the angular dependence of magnetization curves and consequently of the coercive field $\left(H_{C}\right)$ and the saturation one
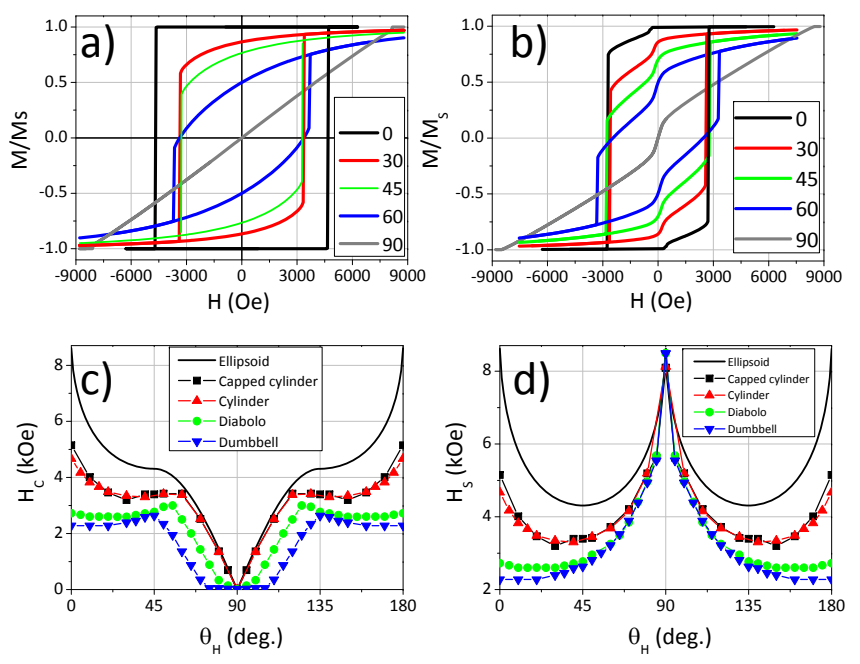

Figure 6: a) Normalized magnetization curves of a cylinder and b) of a diabolo for different $\theta_{H}$ angles. c)d) Angular dependence of $H_{C}$ (and $H_{S}$ ) deduced from the magnetization curves for a cylinder (blue squares), a capped cylinder (green triangles), a diabolo (red circles) and a dumbbell (black circles). The continuous black lines in c) and d) correspond to the ellipsoid case and have been calculated by using equations 4 and 5 . The length of these nano-objects is $L=200$ $\mathrm{nm}$ while the internal diameter is $d=10 \mathrm{~nm}$; in addition, $D$ and $T$ have been fixed to $20 \mathrm{~nm}$ and $10 \mathrm{~nm}$, respectively.

$\left(H_{S}\right)$. These angular dependence of $H_{C}$ and $H_{S}$ are compared to the one coming from the well-known relations for a prolate spheroid object [6, 28]:

$$
H_{C}=2 \pi M_{S}\left(N_{z}-N_{x}\right) \sin 2 \varphi_{H}
$$

and

$$
H_{S}=\frac{4 \pi M_{S}\left(N_{z}-N_{x}\right)}{\left(\sin ^{\frac{2}{3}} \varphi_{H}+\cos ^{\frac{2}{3}} \varphi_{H}\right)^{\frac{3}{2}}} H_{C}
$$

We remained here that $\theta_{H}$ is the angle between the applied magnetic field and the revolution axis of the nanoobjects. In Figure6a) only the cylinder hysteresis normalized loops are presented while in Figure 6b) we reported the diabolo ones. Again, capped cylinder and dumbbell behaviors are not reported because of their great similarity with respectively the cylinder and the diabolo shapes. The cylinder (and capped cylinder) type hysteresis loops show hysteresis behavior very close to the prolate ellipsoid with however lower coercive field values. For the diabolo (and dumbbell) case, hysteresis loops are again much complex and put into evidence the linear dependence before the reversal, characteristics of the non uniform magnetization distribution promoted by the tip. In Figure 6c) and d), we reported the angular dependence of $H_{C}$ and $H_{S}$ given by equations 4 and 5 (for the prolate ellipsoid). It is clear that for both $H_{C}$ and $H_{S}$, in the case of cylinder type nanowires (and capped cylinder), the behavior is very close to the prolate ellipsoid one 
with a minimum at $\varphi=45^{\circ}$ (i.e. as expected from the Stoner-Wohlfarth model). The effect of the morphology (cylinder compared to the prolate ellipsoid) is given by the lower values of both fields which was confirmed also in previous works [30. This drop is, of coarse, more visible when the magnetic field is applied along the nanowires $\left(\varphi_{H}=0^{\circ}\right)$ than when they are close to the perpendicular direction $\left(\varphi_{H}=90^{\circ}\right)$. This is due to the fact that tip effect is less and less "efficient" in the perpendicular configuration. Indeed, the demagnetizing field at the saturation in this case, is uniform and its value is closely to the one of an infinite cylinder with a magnetization at saturation directed perpendicularly to the nanowire length. For the diabolo (and dumbbell), $H_{C}$ et $H_{S}$ are very far from the Stoner-Wohlfarth model. Both fields are much lower than the one from this model, all along the angular dependence. A "plateau-like" behavior is observed for both fields up to a misalignment of 45 degrees. Increasing the misalignment up to the perpendicular configuration $\left(\varphi_{H}=90^{\circ}\right)$, lets both fields behave closely to the other shapes. This is coherent with the fact that tip effect becomes less and less efficient approaching the perpendicular configuration in all different morphologies. All these results are very interesting as they put into evidence that even if the coercivity is not optimized in the case of diabolo and dumbbell nanowires, it is also clear that a higher misalignment is permitted in case of applications. The "plateau-like" region allows, in this latter case, a quite constant value of the coercivity for a few degrees misaligned ensemble of nanowires while this will not be the case for the cylinder type case (i.e. few degrees of misalignment will lower the coercivity of few thousands of $\mathrm{Oe})$.

\section{CONCLUSION}

The static magnetic properties of complex shape Cobalt nanowires have been studied by micromagnetic simulations. The relevance of each characteristic size and their critical values strongly confirms that once the aspect ratio $\left(\frac{L}{d}\right)$ of the nanowire is bigger than around 10 , the length is no more the pertinent parameter and instead the internal diameter and the nanowires tips play a key role. As a consequence, all the experimental effort focusing on improving this aspect ratio value by extending the length of nanowires at a fixed $d$ will be useless in term of shape anisotropy optimization. In addition, angular dependence of magnetization curves reveal that cylinder and capped cylinder shapes behavior (in term of coercive $H_{C}$ and saturating fields $\left.H_{S}\right)$ are close to the "ideal" ellipsoid case (Stoner-Wolfarth model) with however lower $H_{C}$ and $H_{S}$ values. In contrary, diabolo and dumbbell shapes behavior grew apart from this standard model. We attribute these behaviors to the non uniform demagnetizing field distribution which is localized inside the nanowire head (for the whole studied shapes) and which acts as a nucleation point for the magnetization reversal process. In addition, a clear meltdown of the coercive behavior for both dumbbell and diabolo nanowires has been found when increasing the thickness $T$ of the tips. A critical thickness around $T \sim \frac{L}{d}$ is observed for the minimum value of the coercive field in both diabolo and dumbbell geometry. Finally, concerning the potential integration of this kind of nanowires as building bricks for permanent magnet fabrication, we found that a very weak angular dispersion of the nanowires is mandatory to keep a high macroscopic coercive field when using cylinder or capped cylinder nanowires while in case of diabolo and dumbbell nanowires, the "plateau-like" behavior allow strong misalignment.

\section{Acknowledgments}

This work has been partially supported by the Université Paris 13 through a "Bonus Qualité Recherche" project and by the Region Ile-de-France in the framework of C'Nano IdF, the nanoscience competence center of Paris Region (Eco-Nano project).
[1] D. Weller, A. Moser, IEEE Trans. Magn., 35, 4423-4439 (1999)

[2] A. Fert and L. Piraux, J. Mag. Mag. Mater. 200, 228 (1999)

[3] T. Maurer, F. Ott, G. Chaboussant, Y. Soumare, J.-Y. Piquemal and G. Viau, Appl. Phys. Lett. 91, 172501 (2007)

[4] O. Gutfleisch, M. A. Willard, E. Bruck, C. H. Chen, S. G. Sankar and J. Ping Liu, Adv. Mater. , 23, 821-842 (2011)

[5] R. P. Cowburn, J. Phys. D: Appl. Phys. 33, R1-R15 (2000)

[6] S. Bance, J. Fischbacher, T. Schrefl, I. Zins, G. Rieger, C. Cassignol, J. Mag. Mag. Mater. 363, 121-124(2014)

[7] D. J. Sellmyer, M. Zheng and R. Skomski, J. Phys.: Condens. Matter 13, R433 (2001)
[8] T. M. Whitney, P. C. Searson, J. S. Jiang, and C. L. Chien, Science 2611316 (1993)

[9] H. Pan, B. Liu, J. Yi, C. Poh, S. Lim, J. Ding, Y. Feng, C. H. A. Huan and J. Lin, J. Phys. Chem. B 109, 3094 (2005)

[10] X. Y. Zhang, G. H. Wen, Y. F. Chan, R. K. Zheng, X. X. Zhang, and N. Wang, Appl. Phys. Lett. 83, 3341 (2003)

[11] F. Dumestre, B. Chaudret, C. Amiens, M. C. Fromen, M. J. Casanove, P. Renaud and P. Zurcher, Angew. Chem. Int. Ed. 414286 (2002)

[12] Q. Liu, X. Guo, T. Wang, Y. Li, W. Shena, Materials Letters 64 1271-1274 (2010)

[13] D. Ung, G. Viau, C. Ricolleau, F. Warmont, P. Gredin, and F. Fiévet, Adv. Mater., 17, 338 (2005)

[14] D. Ung, Y. Soumare, N. Chakroune, G. Viau, M.-J. Vaulay, V. Richard, and F. Fiévet, Chem. Mater. 19, 
2084 (2007)

[15] Seung I. Cha, Chan B. Mo, Kyung T. Kim, and Soon H. Hong, J. Mater. Res., 20, 2148 (2005)

[16] M. Vazquez, M. Hernandez-Velez, K. Pirota, A. Asenjo, D. Navas, J. Velazquez, P. Vargas and C. Ramos, Eur. Phys. J. B 40, 489 (2004)

[17] Y. Soumare, C. Garcia, T. Maurer, G. Chaboussant, F. Ott, F. Fiévet, J.-Y. Piquemal and G. Viau, Adv. Func. Mater. 19, 1971 (2009)

[18] G. Viau, C. Garcia, T. Maurer, G. Chaboussant, F. Ott, Y. Soumare and J.-Y. Piquemal, Physica Status Solidi (a), 206, 663 (2009)

[19] K. Soulantica, F. Wetz, J. Maynadié, A. Falqui, R. P. Tan, T. Blon, B. Chaudret and M. Respaud, Appl. Phys. Lett. 95, 152504 (2009)

[20] L. G. Vivas, M. Vazquez, J. Escrig, S. Allende, D. Altbir, D. C. Leitao, and J. P. Araujo, Phys. Rev. B 85, 035439 (2012)

[21] V. Sokalski, D. E. Laughlin, and J.-G. Zhu, J. Appl. Phys.
110, $093919(2011)$

[22] F. Ott, J. Appl. Phys. 105, 013915 (2009)

[23] J. Sánchez-Barriga, M. Lucas, F. Radu, E. Martin, M. Multigner, P. Marin, A. Hernando, and G. Rivero, Phys. Rev. B 80, 184424 (2009)

[24] P. E. Tannenwald and R. Weber, Phys. Rev., 121, 715 (1961)

[25] T. Fischbacher, M. Franchin, G. Bordignon and H. Fangohr, IEEE Trans. Mag. 43, 2896 (2007), URL : http://nmag.soton.ac.uk/nmag/

[26] NETGEN, URL: http://www.hpfem.jku.at/netgen/

[27] GMSH, URL: http://geuz.org/gmsh/

[28] L.Sun, Y. Hao, C.-L. Chien and P.-C. Searson, IBM Journal of Research and Development, 49, 1, pg.79 (2005)

[29] P.Krone, D. Makariv, M. Albrecht, T. Schrefl, D. Suess, J. Mag. Mag. Mater. 322, 3771-3776 (2010)

[30] G. Brown, S. M. Stinnett, M. A. Novotny and P. A. Rikvold, J. Appl. Phys. 95, 6666 (2004) 